\title{
Equilibrium strategies in a defined benefit pension plan game
}

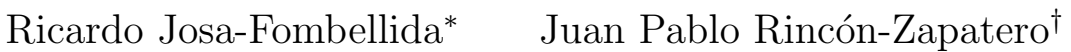

October 10, 2016

\begin{abstract}
We study the optimal management of an aggregated overfunded pension plan of defined benefit type as a two-player noncooperative differential game. The model's key fact is to consider the fund surplus as a strategic variable that makes the pension plan more attractive both for current and future participants. We let the worker participants to act collectively as a single player that claims a share of the surplus, and let the sponsoring firm act as the player that cares about the investment of the surplus fund assets. The union's objective is to maximize the expected discounted utility of the extra benefits claimed. We solve this asymmetric game under two different assumptions on the preferences of the firm: in the first scenario, the firm aims to maximize expected discounted utility derived from fund surplus; while in the second scenario, the firm cares about minimizing the probability that the fund surplus reaches very low values.
\end{abstract}

Keywords: Finance, Pension funding, Portfolio theory, Stochastic differential game, Nash/Pareto equilibria, Dynamic programming.

JEL Classification Numbers: C73, G11, G22.

Mathematics Subject Classification (2010): 91B30, 91G10, 49N90, 91A15.

\section{Introduction}

In defined benefit pension plans, a firm provides benefits to workers when they retire. Benefits have been fixed in advance, and it is the sole responsability of the firm to honor this liability. To this end, a pension plan is designed by using actuarial valuation, which calculates suitable contributions

\footnotetext{
* Departamento de Estadística e Investigación Operativa and IMUVA. Universidad de Valladolid. Paseo de Belén 7, 47011 Valladolid, Spain. ricar@eio.uva.es

${ }^{\dagger}$ Corresponding author. Departamento de Economía. Universidad Carlos III de Madrid. C/ Madrid, 126, 28903 Getafe - Madrid, Spain. jrincon@eco.uc3m.es. Telephone: (34) 9162486 66. Fax: (34) 916249875.
} 
to the fund to meet the promised benefit. Actuarial valuation takes into account such variables as retirement age, employee's life expectancy, mean salary increase, annual retirement benefit amount, worker mobility, the possible evolution of interest rates and stock market performance, among others. Besides contributions made by the employer and possible contributions by the employee, the employer decides how to invest the pension fund's assets.

Pension plans that have more (less) liabilities than assets are known as underfunded (overfunded). That a pension plan becomes under- or overfunded is a dynamic process which depends to a great extent on crashes and booms in the stock market. Whereas, in the underfunded case, it is natural to think that the efforts of the employer should be aimed at minimizing the gap between fund assets and liability, it is not clear what to recommend in the overfunded region. Legislation forbids the distribution of any surplus of the pension plan to shareholders, being devoted to current and future retirees. The firm may simply keep this excess amount in the fund's account, without any other motive than to accumulate assets, or the firm may act more strategically, designing policies that provide, in addtion of safety, additional incentives to future participants to join the pension plan by providing extra rewards to both participants and retirees. On the other hand, workers would surely like to play an active role in taking decisions on how to use the fund surplus. It could be used to lessen the agreed contributions, or to provide an extra benefit - it makes no sense to simply continue accumulating assets to the pension plan if they are not going to be distributed to participants. Moreover, fund assets are subject to taxation. The larger the fund size, the larger the tax payments. Instead of using the fund surplus to pay taxes, agents may wonder why they should not allocate part of this surplus among the participants. As said above, this would make it more attractive for future workers to be hired by the firm and, consequently, enter into the pension plan, making it a stronger financial instrument into the financial market.

The main question we address in this paper is how to manage the surplus when the pension fund is overfunded. In more detail, the objective of the firm to minimize the (squared) difference between fund assets and actuarial liability is fine when the fund is underfunded, but it is not completely satisfactory in the overfunded case, as it refrains from using investment opportunities that may provide high expected returns. To consider quadratic loss functions is common in the literature on the optimal management of defined benefit pension plans; see e.g. Haberman and Sung (1994), Chang (1999), Cairns (2000), Haberman, Butt and Megaloudi (2000), JosaFombellida and Rincón-Zapatero (2001, 2004), Chang et al. (2002, 2003), Delong et al (2008), Xu et al (2007) and Hainaut (2014), among others. Some extensions include stochastic interest rates, as in Huang and Cairns (2006), Josa-Fombellida and Rincón-Zapatero (2010) or Hainaut and Deelstra (2011), or the consideration of jumps in the evolution of benefits and/or 
risky assets, as in Josa-Fombellida and Rincón-Zapatero (2012) or Le Courtois and Menoncin (2015) (the latter in defined contribution pension plans). When the fund is overfunded, the sponsor could face slightly more risky investments to try to raise fund assets ${ }^{1}$. Now, if the firm takes more risky investment decisions, then the participants should be compensated in some way for the exposure of the pension plan to a higher risk of becoming underfunded. We suppose that this compensation is negotiated dynamically between the firm and the representatives of the workers. The compensation consists of a claim on the fund surplus, which can be seen as an excess pension benefit that is added to the agreed benefits at the moment of retirement. No claim of excess benefits is allowed when the pension plan is underfunded. We model this conflict between the firm and the union as a non-zero sum dynamic game. The natural framework is a noncooperative interaction, but we also allow for commitment between players and study cooperative solutions. The main objective of the firms is to maintain fund assets at safe levels so as to be able to pay benefits; however, as this is entirely the employer's responsability, workers may be interested in claiming a share of the excess fund assets in the form of a premium benefit. Nevertheless, there is nothing to withdraw when there is no surplus, so workers have to be careful with their demands in order not to drive the fund into the underfunded region.

To our knowledge, the models that have appeared in the literature of the dynamic management of defined benefit pension plans consider only one decision agent, the sponsor of the plan. The recent paper by Guan and Liang (2016), considers a game between the managers of two defined contribution pension funds, under inflation risks. This game is quite different from the game we propose here. First, our game is for defined benefit plans and second, the conflict arises because the players have different views on how the surplus fund assets should be shared. In the former defined contribution plan game, the conflict comes from two different managers looking for investment opportunities, trying to maximize the expected utility on wealth, by acting non cooperatively. Hence, both players have the same instrument at hand, that is investment. However, in our game, whereas the firm chooses portfolios, the union decides, roughly speaking, about benefits. It is as if in the classical Merton model the consumption/investment decisions were decentralized, with one agent choosing investment and the other choosing consumption (benefits). Hence, the conflict is inside the pension plan itself, and it determines the investment and benefits outcomes of the plan. In

\footnotetext{
${ }^{1}$ This is because, as the fund's assets grow, it could be possible to diminish the planned contributions in the actuarial computations. For instance, if the sponsor is using a spread method of funding, that is, the contribution $C$ equals $N C+j(A L-F)$, where $N C$ is the normal cost, $A L$ is the liability, $F$ fund's assets, and $j>0$ is the velocity of adjustment of the contribution to the normal cost, then contributions are below the normal cost in the overfunded region.
} 
Guan and Liang (2016), as well as in the rest of references below dealing with stochastic portfolio games, the interaction is between two different pension plans (or investment managers, depending of the context). The structure of the game is also different from other portfolio games that have appeared in the literature. It is worth mentioning Browne (2000), where a variety of games that model different preferences of the agents are considered: utility maximization, ruin probability minimization and minimizing/maximizingexpected-time to reach an objective. The game that we propose in this paper has elements in common with Leong and Huang (2010), which studies an stochastic version of the differential game of capitalism in Lancaster (1973), where the players are the government, that maximizes a vote function, and a representative firm, that maximizes a flow of dividend payments for the shareholders, or with Cabo and García-González (2014). where the authors consider, in a deterministic setting, a dynamic game of public pension provision between the government and a representative employee, in order to determine the optimal retirement age. The players have quadratic preferences.

We find that players with CRRA utilities use, in equilibrium, linear strategies, proportional to the fund surplus, in such a way that the fund surplus never becomes null with positive probability in finite time. Moreover, when the players have the same relative risk aversion coefficient, the Nash equilibrium is Pareto optimal. Also, we show that if the aim of the firm is to minimize the probability of ruin, this objective is attained for a firm that wishes to maximize the utility obtained from the fund's assets surplus for a specific relative risk aversion coefficient. Overall, what we do is to design a scheme of how the fund could be split between the participants and the firm when the pension plan is overfunded. This division is not based on commitment, and no player has an incentive to deviate from this recommendation, even at any intermediate stage of the interaction.

The paper is organized as follows. In Section 2. we define the elements of the pension plan and the financial market. We let the worker participants to act collectively as a single player that claims a share of the surplus, and let the sponsoring firm act as the player that cares about the investment of the surplus fund assets. The union's objective is to maximize the expected discounted utility of the extra benefits claimed. We solve the game under two different assumptions on the preferences of the firm: while in the first scenario, the firm aims to maximize expected discounted utility derived from fund surplus; in the second scenario, considered in Section 3, the firm cares about minimizing the probability that the fund surplus should reach very low values. Finally, Section 4 establishes some further research directions. 


\section{The pension game}

In this section, we set the elements of the maiden game: the financial market, the fund surplus evolution and the payoffs and strategies of the players. After solving for the Nash equilibrium, we show that it is also a Pareto outcome when the players have the same discount factors and the same risk aversion coefficients.

\subsection{Financial market and fund surplus evolution}

The financial market is that introduced by Merton (1971). Consider a probability space $(\Omega, \mathscr{F}, \mathbb{P})$, where $\mathbb{P}$ is a probability measure on $\Omega$ and $\mathscr{F}=\left\{\mathscr{F}_{t}\right\}_{t \geq 0}$ is a complete and right continuous filtration generated by the $n$-dimensional standard Brownian motion $w=\left(w_{1}, \ldots, w_{n}\right)^{\top}$, that is, $\mathscr{F}_{t}=\sigma\left\{w_{1}(s), \ldots, w_{n}(s) ; 0 \leq s \leq t\right\}$.

As in Merton's model, the investor may trade between a riskless bond $S^{0}$ and $n$ risky assets $S^{1}, \ldots, S^{n}$, which are geometric Brownian motions.

$$
\begin{aligned}
& d S^{0}(t)=r S^{0}(t) d t, \quad S^{0}(0)=1, \\
& d S^{i}(t)=S^{i}(t)\left(b_{i} d t+\sum_{j=1}^{n} \sigma_{i j} d w_{j}(t)\right), \quad S^{i}(0)=s_{i}, \quad i=1, \ldots, n .
\end{aligned}
$$

The constant $r>0$ denotes the short risk-free rate of interest, $b_{i}>0$ the mean rate of return of the risky asset $S^{i}$, and $\sigma_{i j}>0$ the volatility coefficients. It is assumed that $b_{i}>r$, for each $i=1, \ldots, n$, so the investor has incentives to invest with risk. The matrix $\left(\sigma_{i j}\right)$ is denoted by $\sigma$ and the Sharpe ratio or market price of risk for this portfolio, $\sigma^{-1}(b-r \overline{1})$, by $\theta$, where $b=\left(b_{1}, \ldots, b_{n}\right)^{\top}$ and $\overline{1}$ is a (column) vector of ones. The market is complete, that is, the symmetric matrix $\Sigma=\sigma \sigma^{\top}$ is positive definite.

In our framework, the investor is the firm sponsoring the pension plan, who at time $t=0$ possesses an initial endowment of fund reserves above the liability. We denote by $X(t)$ the surplus at time $t$. At the initial time, this excess value is $X(0)=x>0$. Whenever $X$ is positive, the union claims a share of $X$ in the form of extra benefits. No claim is admitted if $X$ becomes zero or negative. The sponsoring firm forms portfolios dynamically with the bond and the risky assets, trading among them. Let $\pi_{i}$ be the chosen amount of surplus to be invested in the asset $i \in\{1, \ldots, n\}$, and let $\pi_{0}$ be the amount invested in the bond; clearly $\pi_{0}=X-\sum_{i=1}^{n} \pi_{i}$. We denote by $P$ the extra benefits claimed by the union. Under the trading strategy $\pi=\left(\pi_{1}, \ldots, \pi_{n}\right)^{\top}$ and benefits $P, X$ follows the dynamics

$$
d X(t)=\sum_{i=1}^{n} \pi_{i}(t) \frac{d S^{i}(t)}{S^{i}(t)}+\left(X-\sum_{i=1}^{n} \pi_{i}(t)\right) \frac{d S^{0}(t)}{S^{0}(t)}-P(t) d t
$$


or, after substituting (1) and (2) in (3)

$$
d X(t)=\left(r X(t)+\pi^{\top}(t)(b-r \overline{1})-P(t)\right) d t+\pi^{\top}(t) \sigma d w(t),
$$

which, toghether with the initial condition $X(0)=x$, drives the asset's surplus fund evolution.

Now we define the class of admissible strategies of the union, $\mathcal{A}^{U}$, and the firm, $\mathcal{A}^{F}$. The union chooses benefits and the firm chooses the portfolio. Investment and benefit strategies are both Markovian and stationary, that is, they depend only on the state variable $x$ and are independent of time, $\pi \equiv \pi(x), P \equiv P(x)$. As said above, benefits cannot take on negative values, but they are null if the pension plan gets underfunded, thus $P(x) \geq 0$ for any $x>0$ and $P(x)=0$ if $x \leq 0$. We also assume that both processes $\{\pi(X(t)): t \geq 0\}$ and $\{P(X(t)): t \geq 0\}$ are adapted to the filtration $\left\{\mathscr{F}_{t}\right\}_{t \geq 0}$ and $\mathscr{F}_{t}-$ measurable. Moreover, $(P, \pi)$ satisfies the integrability condition

$$
\mathbb{E} \int_{0}^{T} P(t) d t+\mathbb{E} \int_{0}^{T} \pi^{\top}(t) \pi(t) d t<\infty, \quad \forall T>0 .
$$

Then, given $X(0)=x,(4)$ admits a unique strong solution. Note that in (5) we are identifying $\pi(t)$ with $\pi(X(t))$, as well as $P(t)$ with $P(X(t))$, to shorten notation.

\subsection{Players' Payoffs}

Let the strategic profile $(P, \pi) \in \mathcal{A}^{U} \times \mathcal{A}^{M}$. Given $\pi$, the union chooses $P$ that maximizes the payoff

$$
J_{U}(x ; P, \pi)=\mathbb{E}_{x} \int_{0}^{\infty} e^{-\alpha t} u(P(t)) d t,
$$

where $u$ is a utility function of benefits and $\alpha>0$ is the time preference of the union.

In the same way, given $P$, the firm seeks $\pi$ to maximize the payoff

$$
J_{F}(x ; P, \pi)=\mathbb{E}_{x} \int_{0}^{\infty} e^{-\beta t} v(X(t)) d t,
$$

where $v$ is a utility function of the fund surplus and $\beta>0$ is the time preference of the firm.

To obtain explicit solutions, we focus on CRRA utility functions ${ }^{2}$ :

$$
\begin{array}{ll}
u(P)=\frac{P^{1-\gamma}}{1-\gamma}, & 0<\gamma<1, \\
v(X)=\frac{X^{1-\delta}}{1-\delta}, & 0<\delta<1 .
\end{array}
$$

\footnotetext{
${ }^{2}$ When $\gamma=1$ and $\delta=1$, the utility functions are type: $u(P)=\ln P, v(X)=\ln X$.
} 
We assume that the risk aversion indexes $\gamma, \delta$ are in $(0,1)$, so the utility functions are both strictly concave.

\subsection{Nash equilibrium strategies}

In a dynamic noncooperative setting, the relevant solution concept is the Markov perfect Nash equilibrium. An MPNE of the pension game is a pair of admissible strategies $\left(P^{*}, \pi^{*}\right) \in \mathcal{A}^{U} \times \mathcal{A}^{F}$ such that, for any $(P, \pi) \in$ $\mathcal{A}^{U} \times \mathcal{A}^{F}$, for any $x>0$

$$
\begin{aligned}
& J_{U}\left(x ; P^{*}, \pi^{*}\right) \geq J_{U}\left(x ; P, \pi^{*}\right), \\
& J_{F}\left(x ; P^{*}, \pi^{*}\right) \geq J_{F}\left(x ; P^{*}, \pi\right) .
\end{aligned}
$$

Let $V_{U}$ and $V_{F}$ be the value function of the union and the firm respectively, that is

$$
\begin{aligned}
& V_{U}(x)=J_{U}\left(x ; P^{*}, \pi^{*}\right), \\
& V_{F}(x)=J_{F}\left(x ; P^{*}, \pi^{*}\right) .
\end{aligned}
$$

In the next proposition, we state the form of the MPNE of the pension game.

Proposition 2.1 Assume that the parameters of the game are such that the constants A, B given in (8) and (9) below are both positive. Then the value function of the union and the firm in the Nash pension game (4), (6), (7) are

$$
\begin{aligned}
& V_{U}(x)=A \frac{x^{1-\gamma}}{1-\gamma} \\
& V_{F}(x)=B \frac{x^{1-\delta}}{1-\delta}
\end{aligned}
$$

respectively, where

$$
\begin{aligned}
A & =\left(\frac{\alpha}{\gamma}-\frac{1-\gamma}{\gamma}\left(r+\left(\frac{1}{\delta}-\frac{\gamma}{2 \delta^{2}}\right) \theta^{\top} \theta\right)\right)^{-\gamma} \\
B & =\frac{1}{1-\delta}\left(\frac{\beta}{1-\delta}+\frac{\alpha-r}{\gamma}+\left(\frac{1-\gamma}{2 \delta^{2}}-\frac{1-\gamma}{\gamma \delta}-\frac{1}{2 \delta}\right) \theta^{\top} \theta\right)^{-1}
\end{aligned}
$$

The MPNE $\left(P^{*}, \pi^{*}\right)$ is given by

$$
\begin{aligned}
& P^{*}(x)=A^{-1 / \gamma} x, \\
& \pi^{*}(x)=\frac{1}{\delta} \Sigma^{-1}(b-r \overline{1}) x
\end{aligned}
$$


and the equilibrium fund surplus is a geometric Brownian motion process,

$$
d X^{*}(t)=X^{*}(t)\left(r+\frac{1}{\delta} \theta^{\top} \theta-A^{-1 / \gamma}\right) d t+X^{*}(t) \frac{1}{\delta} \theta^{\top} d w(t),
$$

thus $X^{*}>0$.

Proof. The Hamilton-Jacobi-Bellman system of PDEs of the Nash pension game is

$$
\begin{aligned}
& \alpha V_{U}(x)=\max _{P}\left\{\frac{P^{1-\gamma}}{1-\gamma}+\left(r x+\pi^{\top}(b-r \overline{1})-P\right) V_{U}^{\prime}(x)\right\}+\frac{1}{2} \pi^{\top} \Sigma \pi V_{U}^{\prime \prime}(x), \\
& \beta V_{F}(x)=\max _{\pi}\left\{\frac{x^{1-\delta}}{1-\delta}+\left(r x+\pi^{\top}(b-r \overline{1})-P\right) V_{F}^{\prime}(x)+\frac{1}{2} \pi^{\top} \Sigma \pi V_{F}^{\prime \prime}(x)\right\} .
\end{aligned}
$$

From the optimality conditions we have

$$
\begin{gathered}
P^{-\gamma}-V_{U}^{\prime}(x)=0 \Rightarrow P=\left(V_{U}^{\prime}(x)\right)^{-1 / \gamma} \\
(b-r \overline{1}) V_{F}^{\prime}(x)+\sigma \sigma^{\top} \pi V_{F}^{\prime \prime}(x)=0 \Rightarrow \pi=-\Sigma^{-1}(b-r \overline{1}) \frac{V_{F}^{\prime}(x)}{V_{F}^{\prime \prime}(x)} .
\end{gathered}
$$

Plugging these expressions into the HJB system, and after some simplifications, we have

$$
\left\{\begin{array}{l}
\alpha V_{U}(x)=\frac{\gamma}{1-\gamma}\left(V_{U}^{\prime}(x)\right)^{1-1 / \gamma}+r x V_{U}^{\prime}(x)-\theta^{\top} \theta \frac{V_{F}^{\prime}(x)}{V_{F}^{\prime \prime}(x)} V_{U}^{\prime}(x)+\frac{1}{2} \theta^{\top} \theta\left(\frac{V_{F}^{\prime}(x)}{V_{F}^{\prime \prime}(x)}\right)^{2} V_{U}^{\prime \prime}(x), \\
\beta V_{F}(x)=\frac{x^{1-\delta}}{1-\delta}+r x V_{F}^{\prime}(x)-\frac{1}{2} \theta^{\top} \theta \frac{\left(V_{F}^{\prime}(x)\right)^{2}}{V_{F}^{\prime \prime}(x)}-\left(V_{U}^{\prime}(x)\right)^{-1 / \gamma} V_{F}^{\prime}(x) .
\end{array}\right.
$$

Let us try solutions $V_{U}(x)=A \frac{x^{1-\gamma}}{1-\gamma}, V_{F}(x)=B \frac{x^{1-\delta}}{1-\delta}$, where $A, B>0$ are suitable constants that are determined with the help of the equations (15). After substituting the guessed value functions, we obtain homogeneous expressions in the powers $x^{1-\gamma}$ and $x^{1-\delta}$, respectively. Thus, the following two algebraic equations for $A, B$ have to be fulfilled

$$
\begin{aligned}
& \frac{\alpha}{1-\gamma} A=\frac{\gamma}{1-\gamma} A^{1-1 / \gamma}+r A+\frac{1}{\delta} \theta^{\top} \theta A-\frac{\gamma}{2 \delta^{2}} \theta^{\top} \theta A, \\
& \frac{\beta}{1-\delta} B=\frac{1}{1-\delta}+r B+\frac{1}{2 \delta} \theta^{\top} \theta B-A^{-1 / \gamma} B .
\end{aligned}
$$

Solving for $A$ and $B$ to get (8) and (9) is straightforward.

Now, the equilibrium candidate (10) and (11) is obtained from the optimality conditions (13) and (14), once $V_{U}$ and $V_{F}$ have been substituted. To see that the pair $\left(P^{*}, \pi^{*}\right)$ is indeed an MPNE, note that $V_{F}^{\prime \prime}<0$, because $B>0$, thus $\pi^{*}$ is a true maximizer of the HJB equation for the firm-it was already clear that $P^{*}$ was a maximizer of the HJB equation of the union-. 
Upon substitution of $\left(P^{*}, \pi^{*}\right)$ into the $\operatorname{SDE}(4)$, we get $(12)$, thus $\left(P^{*}, \pi^{*}\right)$ is clearly an admissible pair of strategies. In fact, the solution of (12) is

$$
X^{*}(t)=x \exp \left(\left(r+\frac{1}{\delta} \theta^{\top} \theta-A^{-1 / \gamma}-\frac{1}{2 \delta^{2}} \theta^{\top} \theta\right) t+\frac{1}{\delta} \theta^{\top} \int_{0}^{t} w(s) d s\right),
$$

hence $X^{*}(t)>0$. Finally, we prove that the transversality condition holds, that is

$$
\lim _{t \rightarrow \infty} e^{-\alpha t} \mathbb{E}_{x} V_{U}\left(X^{*}(t)\right)=\lim _{t \rightarrow \infty} e^{-\beta t} \mathbb{E}_{x} V_{F}\left(X^{*}(t)\right)=0 .
$$

See, for instance, Hernández-Lerma (1994), Section 7, or Theorem 8.5 of Dockner et al (2000). By Arnold (1974), p. 139, for any real number $a$,

$$
\mathbb{E}_{x}\left(X^{*}(t)\right)^{a}=x^{a} \exp \left\{a\left(r+\frac{1}{\delta} \theta^{\top} \theta-A^{-1 / \gamma}-\frac{1}{2 \delta^{2}} \theta^{\top} \theta\right) t+\frac{a^{2}}{2 \delta^{2}} \theta^{\top} \theta t\right\} .
$$

After replacing $a$ by $1-\gamma$ and then for $1-\delta$, we see that (16) holds if and only if

$$
\begin{aligned}
(1-\gamma)\left(r+\frac{1}{\delta} \theta^{\top} \theta-A^{-1 / \gamma}-\frac{1}{2} \frac{\gamma}{\delta^{2}} \theta^{\top} \theta\right) & <\alpha, \\
(1-\delta)\left(r-A^{-1 / \gamma}+\frac{1}{2 \delta} \theta^{\top} \theta\right) & <\beta
\end{aligned}
$$

These two conditions are equivalent to the nonnegativity of the constants $A>0$ and $B>0$ respectively, which is assumed in the proposition.

Remark 2.1 (Study of the signs of $A$ and $B$ ) Positivity of the constants $A$ and $B$ are crucial in the above result. Note that by no means do $A$ and/or $B$ negative imply that a Nash equilibrium does not exist, only that it is not in linear strategies. To explore if the game admits a Nash equilibrium out of the conditions imposed in the result above is beyond the scope of this paper. The constant $A$ defined in (8) is positive if and only if $\alpha>(1-\gamma)\left(r+f(\delta) \theta^{\top} \theta\right)$, where $f(\delta)=\frac{1}{\delta}-\frac{\gamma}{2 \delta^{2}}$. It is clear that the more risk averse the players are (i.e., $\delta, \gamma \rightarrow 1$, logarithmic utility), the easier it is for the inequality to be fulfilled. On the contrary, for players showing very small risk aversion (i.e., $\delta, \gamma \rightarrow 0)$, the inequality may require high discount factors of the union. Analytically, this is because $f$ attains its maximum value at $\delta=\gamma$, hence players having the same risk aversion is the most unfavorable case, and in this case $\alpha$ should be greater than $(1-\gamma)\left(r+\frac{1}{2 \gamma} \theta^{\top} \theta\right)$. Obviously, small values of $\gamma$ would need large values of the discount factor $\alpha$. Analogously, the constant $B$ is positive if and only if $\beta>\frac{1-\delta}{\gamma}(r-\alpha+(1-\gamma) g(\delta))$, where $g(\delta)=\frac{1}{\delta}-\frac{\gamma}{2 \delta^{2}}+\frac{\gamma}{2 \delta(1-\gamma)}$. We have a completely similar situation. The inequality is more likely to be fulfilled as the risk aversion is higher. Now, players close to being risk neutral would imply high discount factors $\beta$ of the firm. 
Remark 2.2 The previous proposition remains true for logarithmic utility functions $u(P)=\ln P$ and $v(X)=\ln X$. The value functions of the union and the firm are

$$
\begin{aligned}
& V_{U}(x)=\frac{1}{\alpha} \ln x+\frac{1}{\alpha^{2}}\left(r+\frac{\theta^{\top} \theta}{2}\right)+\frac{1}{\alpha}(\ln \alpha-1), \\
& V_{F}(x)=\frac{1}{\beta} \ln x+\frac{1}{\beta^{2}}\left(r+\frac{\theta^{\top} \theta}{2}\right)-\frac{\alpha}{\beta^{2}},
\end{aligned}
$$

respectively, the MPNE is given by $P^{*}(x)=\alpha x, \pi^{*}(x)=\Sigma^{-1}(b-r \overline{1}) x$, and the equilibrium fund surplus is the geometric Brownian motion process,

$$
d X^{*}(t)=\left(r+\theta^{\top} \theta-\alpha\right) X^{*}(t) d t+\theta^{\top} X^{*}(t) d w(t) .
$$

Note that the transversality condition is easily fulfilled because $\mathbb{E}_{x} \ln X^{*}(t)=$ $\ln x+\left(r+\theta^{\top} \theta-\alpha\right) t$.

\subsubsection{Comparative statics of Nash equilibrium}

Both equilibrium strategies found in Proposition 2.1 are linear in fund surplus. The firm investment strategy, $\pi^{*}(x)=\frac{1}{\delta} \Sigma^{-1}(b-r \overline{1}) x$, is also the optimal investment strategy for the Merton model of maximizing the utility from consumption with a CRRA utility function (of course, the original Merton model is not a two-player game). Note that, in the pension game, maximization is performed with respect to fund surplus (or wealth). The expression of $\pi^{*}$ depends on the firm risk aversion coefficient, $\delta$, the excess mean returns of the risky assets with respect to the bond, $(b-r \overline{1})$, and on the inverse of the matrix $\Sigma$, which collects the volatility coefficients of the risky assets. For $\delta=1$, i.e., when the utility is logarithmic, (1990, Ch. 6) demonstrates that $\pi^{*}$ maximizes the discounted and expected rate at which the fund surplus (or wealth) compounds. For this reason, it is called the optimal-growth portfolio strategy. For the very same reason, $\pi^{*}$ minimizes the expected time to hit a higher fund (or wealth) prescribed level. Hence, in equilibrium, the firm aims to maximize the fund growth rate, according to the attitude towards risk shown in the firm's preferences. This is, of course, an objective that is aligned with the union's preferences, as it is obvious that the greater the growth of the fund, the greater the benefits that the workers will claim. In fact, we will prove in Section 2.4 that, when both players have the same risk aversion coefficient, the MPNE is Pareto efficient. Finally, note that the investment strategy, $\pi^{*}$, decreases with the risk aversion coefficient of the firm, $\delta$. On the other hand, the union's equilibrium strategy, $P^{*}(x)=A^{-1 / \gamma} x$, shows a more complicated dependence on the parameters of the pension game. The constant $A$ depends on the attitude towards risk of both players, the subjective discount factor of the union of workers, and finally, elements of the financial market that summarize the risk and return 
of the assets. Computing the derivatives of the constant of proportionality $A^{-1 / \gamma}=\frac{1-\gamma}{\gamma}\left(\frac{\alpha}{1-\gamma}-r-\frac{1}{\delta} \theta^{\top} \theta+\frac{\gamma}{2 \delta^{2}} \theta^{\top} \theta\right)$ is easy. We have

$$
\begin{aligned}
& \frac{\partial A^{-1 / \gamma}}{\partial \alpha}=\frac{1}{\gamma} \\
& \frac{\partial A^{-1 / \gamma}}{\partial r}=-\frac{1-\gamma}{\gamma} \\
& \frac{\partial A^{-1 / \gamma}}{\partial \theta}=\frac{(1-\gamma)(\gamma-2 \delta)}{\delta^{2} \gamma} \theta^{\top}, \\
& \frac{\partial A^{-1 / \gamma}}{\partial \delta}=\frac{(1-\gamma)(\delta-\gamma)}{\delta^{3} \gamma} \theta^{\top} \theta \quad(\delta \neq \gamma), \\
& \frac{\partial A^{-1 / \gamma}}{\partial \gamma}=-\frac{\gamma-2 \delta}{2 \delta^{2} \gamma} \theta^{\top} \theta-\frac{\alpha-r}{\gamma} \quad(\gamma \neq \delta) .
\end{aligned}
$$

As expected, (17) shows that $P^{*}$ increases with $\alpha$, since the union cares more about the present than about the future when the subjective discount factor increases. Expression (18) shows a negative dependence of $P^{*}$ with respect to the riskless rate of return, $r$, since $0<\gamma<1$. This is because the larger $r$ is, the larger the return of the portfolio, all the other elements being constant. Hence it pays to withdraw less surplus now in order to get a larger average return in the future, so the total expected benefit collected will be greater. The influence of the Sharpe ratio on benefits appears in (19) and depends on the relative size of the relative risk aversion coefficient of the players. The benefits are increasing (decreasing) if $\gamma>2 \delta(\gamma<2 \delta)$, that is, if the risk aversion of the union is more (less) than the double of the risk aversion of the manager. In the particular case $\gamma=2 \delta$, benefits are independent of the Sharpe ratio.

In (20), we have that benefits decrease when the risk aversion parameter of the manager increases and the manager is more risk averse than the union, but $P^{*}$ increases when the union is more risk averse than the firm. The benefits $P^{*}$ reaches its minimum value when both union and firm have the same risk aversion index, $\delta=\gamma$, and it is $P^{*}(x)=\frac{1-\gamma}{\gamma}\left(\frac{\alpha}{1-\gamma}-r-\frac{\theta^{\top} \theta}{2 \gamma}\right) x$.

The dependence on its own risk aversion parameter is given in (21) and this shows many possibilities. If the discount rate $\alpha$ is greater than $r-\frac{1}{\delta} \theta^{\top} \theta$, then the benefits are decreasing with $\gamma$. This is because uncertainty makes the union save more now to get greater surplus in the future; this behavior is speeded up because the union cares more about the present than the future. However, if $\alpha$ is not large enough, we can find regions for which benefits increase as $\gamma$ decreases. In particular, this happens for $\gamma$ close to 0 (when the union is "almost" risk neutral). 


\subsubsection{Surplus and extra benefits equilibrium evolution}

In order to study the expected surplus evolution, we take expectation in (12) to obtain

$$
\mathbb{E}_{x} X(t)=x \exp \left\{\left(r+\frac{1}{\delta} \theta^{\top} \theta-A^{-1 / \gamma}\right) t\right\}
$$

that converges to $\infty$ if $r+\frac{1}{\delta} \theta^{\top} \theta>A^{-1 / \gamma}$, that is to say, when $r-\alpha+\frac{1}{\delta} \theta^{\top} \theta>$ $\frac{1}{2 \delta^{2}} \gamma(1-\gamma) \theta^{\top} \theta$, and converges to 0 , otherwise.

From (12), we also can obtain the benefits evolution, using $P^{*}=A^{-1 / \gamma} X$ :

$$
d P(t)=\left(r+\frac{1}{\delta} \theta^{\top} \theta-A^{-1 / \gamma}\right) P(t) d t+\frac{1}{\delta} \theta^{\top} P(t) d w(t),
$$

$P(0)=A^{-1 / \gamma} x$. The expected benefits can be obtained easily, given $X(0)=$ $x$ :

$$
\mathbb{E}_{x} P^{*}(t)=A^{-1 / \delta} \mathbb{E}_{x} X(t)=A^{-1 / \delta} x e^{\left(r+\frac{1}{\delta} \theta^{\top} \theta-A^{-1 / \gamma}\right) t} .
$$

\subsection{Pareto equilibrium strategies}

Suppose now that the players coordinate efforts to attain an efficient solution. In a two-player game, a Pareto equilibrium is a cooperative solution of the game in which there is no possibility of improving the payoff of one of the players without diminishing the payoff of the other player. Thus, $\left(P^{c}, \pi^{c}\right)$ is a Pareto equilibrium if there is no admissible $(P, \pi)$ such that

$$
\begin{aligned}
& J_{U}(x ; P, \pi) \geq J_{U}\left(x ; P^{c}, \pi^{c}\right), \\
& J_{F}(x ; P, \pi) \geq J_{F}\left(x ; P^{c}, \pi^{c}\right),
\end{aligned}
$$

with at least one of the inequalities above being strict. It is well known that with suitable convexity, as is the case in our framework, Pareto equilibrium can be obtained by solving a control problem where the dynamics remains the same as in the Nash game, but the payoff is a convex combination of the players' payoffs. Let us denote the weight in the convex combination by $\mu$. As $\mu$ takes values in the interval $[0,1]$, different Pareto equilibria are obtained. Under the assumption that both players have the same negotiation power, it is common to take $\mu=1 / 2$. We will find, however, the Pareto equilibrium for any $\mu$.

Hence the payoff to be maximized on the class of the admissible controls $\mathcal{A}^{U} \times \mathcal{A}^{F}$ is:

$$
J_{\mu}(x ; P, \pi)=\mathbb{E}_{x} \int_{0}^{\infty}\left(\mu e^{-\alpha t} u(P(t))+(1-\mu) e^{-\beta t} v(X(t))\right) d t
$$

where $u$ and $v$ are utility functions of benefits and surplus, respectively, $\alpha>$ 0 and $\beta>0$ are the time preference of the union and the sponsor, and $\mu \in$ 
$[0,1]$ is a parameter. As well known, $\alpha \neq \beta$ leads to a problem with temporal inconsistence. We want to avoid this complications and focus on the timeconsistent case to compare with the MPNE found in the previous section. Moreover, an explicit solution is beyond our efforts in the asymmetric game $\gamma \neq \delta$. Again, in order to get a neat solutions easily comparable with the outcome of the nooncooperative game, we focus on the case where the CRRA utilities have the same relative risk aversion parameter. Thus, we assume $\alpha=\beta=\rho$ and $\gamma=\delta$, so the payoff becomes

$$
J_{\mu}(x ; P, \pi)=\mathbb{E}_{x} \int_{0}^{\infty} e^{-\rho t}\left(\mu \frac{P(t)^{1-\gamma}}{1-\gamma}+(1-\mu) \frac{X(t)^{1-\gamma}}{1-\gamma}\right) d t .
$$

In order to solve the game with the dynamic programming approach (see for instance Dockner et al (2000)), we obtain the HJB equations. Let $W$ be the value function,

$$
W(x)=\max _{(P, \pi) \in \mathcal{A}^{U} \times \mathcal{A}^{F}}\left\{J_{\mu}(x ; P, \pi) \text { : s.t. }(4)\right\} .
$$

We use the following notation to simplify the exposition. Let

$$
\begin{aligned}
m & =\frac{\rho}{1-\gamma}-r-\frac{1}{2 \gamma} \theta^{\top} \theta, \\
n & =\frac{1-\mu}{1-\gamma} \\
\tilde{n} & =\frac{\gamma}{1-\gamma} \mu^{1 / \gamma} .
\end{aligned}
$$

Note that $n, \tilde{n}>0$.

Proposition 2.2 Assume that $m>0$. Then the value function of the control problem (4), (22), is

$$
W(x)=K \frac{x^{1-\gamma}}{1-\gamma},
$$

where the constant $K$ is the unique positive solution of

$$
\left(\frac{\rho}{1-\gamma}-r-\frac{1}{2 \gamma} \theta^{\top} \theta\right) K^{1 / \gamma}-\frac{1-\mu}{1-\gamma} K^{1 / \gamma-1}-\frac{\gamma}{1-\gamma} \mu^{1 / \gamma}=0,
$$

the Markov Pareto equilibrium is

$$
\begin{aligned}
P^{c}(x) & =\mu^{1 / \gamma} K^{-1 / \gamma} x, \\
\pi^{c}(x) & =\frac{1}{\gamma} \Sigma^{-1}(b-r \overline{1}) x,
\end{aligned}
$$

and the equilibrium fund surplus evolution is given by

$$
d X^{*}(t)=X^{*}(t)\left(r+\frac{1}{\gamma} \theta^{\top} \theta-\left(\frac{K}{\mu}\right)^{-1 / \gamma}\right) d t+X^{*}(t) \frac{1}{\gamma} \theta^{\top} d w(t)
$$


Proof. The HJB equation is

$$
\rho W(x)=\max _{P, \pi}\left\{\mu \frac{P^{1-\gamma}}{1-\gamma}+(1-\mu) \frac{x^{1-\gamma}}{1-\gamma}+\left(r x+\pi^{\top}(b-r \overline{1})-P\right) W^{\prime}(x)+\frac{1}{2} \pi^{\top} \sigma \sigma^{\top} \pi W^{\prime \prime}(x)\right\} .
$$

From the optimality conditions one gets

$$
\begin{gathered}
\mu P^{-\gamma}-W^{\prime}(x)=0 \Rightarrow P=\left(\frac{W^{\prime}(x)}{\mu}\right)^{-1 / \gamma}, \\
(b-r \overline{1}) W^{\prime}(x)+\sigma \sigma^{\top} \pi W^{\prime \prime}(x) \Rightarrow \pi=-\Sigma^{-1}(b-r \overline{1}) \frac{W^{\prime}(x)}{W^{\prime \prime}(x)} .
\end{gathered}
$$

After plugging these expression into the HJB equation above and collecting some terms we lead to the PDE

$$
\rho W(x)=\frac{\gamma \mu^{1 / \gamma}}{1-\gamma}\left(W^{\prime}(x)\right)^{1-1 / \gamma}+\frac{1-\mu}{1-\gamma} x^{1-\gamma}+r x W^{\prime}(x)-\frac{1}{2} \theta^{\top} \theta \frac{\left(W^{\prime}(x)\right)^{2}}{W^{\prime \prime}(x)} .
$$

Let us try $W(x)=K \frac{x^{1-\gamma}}{1-\gamma}$, with $K>0$ a suitable constant. By substituting, the HJB equation is satisfied if $K$ is a positive solution to the algebraic equation (23). Let us show that this equation admits a unique positive solution $\widetilde{K}$. Let $f(K)=m K^{1 / \gamma}-n K^{1 / \gamma-1}-\tilde{n}$ be the function defined by the left hand side of the equality. This function is continuous on $[0, \infty)$ since $0<\gamma<1$. Moreover, $m, n, \tilde{n}>0$ by assumption, hence $f(0)<0$. Computing the derivative of $f$ with respect to $K$ we have that $f$ is decreasing in $\left[0, \frac{(1-\gamma) n}{m}\right]$ and increasing in $\left[\frac{(1-\gamma) n}{m}, \infty\right)$, with $\lim _{K \rightarrow \infty} f(K)=\infty$, thus $f(K)=0$ admits a unique solution. Now, we proceed to check that the coefficient or constant of proportionality defining $p^{c}(x)$, which can be written $\frac{\gamma}{1-\gamma}\left(m-n K^{-1}\right)$, is positive. This is true if and only if the zero of $f, K$, is greater than $\frac{n}{m}$. To see that this is indeed the case, note that $f\left(\frac{n}{m}\right)=$ $m\left(\frac{n}{m}\right)^{1 / \gamma}-n\left(\frac{n}{m}\right)^{1 / \gamma-1}-\tilde{n}=-\tilde{n}<0$, thus $\frac{n}{m}<K$ since $f$ is negative to the left of $K$ and positive to the right.

Finally, we complete the proof by showing that the transversality condition holds. By substituting the expression for $W$ into the optimality conditions, (27) and (28), we obtain (24) and also (25). Again, by HernándezLerma (1994) Section 7, or Theorem 8.5 of Dockner et al (2000), the proof concludes when the transversality condition is checked:

$$
\lim _{t \rightarrow \infty} e^{-\rho t} \mathbb{E}_{x} W\left(X^{*}(t)\right)=0
$$

As the surplus evolution with the optimal strategies is given by (26), thus, by Arnold (1974), p. 139, for a real number $a$,

$$
\mathbb{E}_{x}\left(X^{*}(t)\right)^{a}=x^{a} \exp \left\{a\left(r+\frac{1}{\gamma} \theta^{\top} \theta-\left(\frac{K}{\mu}\right)^{-1 / \gamma}-\frac{1}{2} \frac{\theta^{\top} \theta}{\gamma^{2}}\right) t+\frac{a^{2}}{2} \frac{\theta^{\top} \theta}{\gamma^{2}} t\right\} .
$$


As in the proof of Proposition 2.1, after replacing $a$ by $1-\gamma$, we see that (29) holds if and only if

$$
(1-\gamma)\left(r+\frac{1}{\gamma} \theta^{\top} \theta-\mu^{1 / \gamma} K^{-1 / \gamma}-\frac{1}{2 \gamma} \theta^{\top} \theta\right)<\rho .
$$

By (23), this condition is equivalent to

$$
\frac{1-\gamma}{\gamma}\left(r-\frac{\rho}{1-\gamma}+\frac{1}{2 \gamma} \theta^{\top} \theta+(1-\mu) K^{-1}\right)<0
$$

This is true because $K>(1-\gamma) n / m=(1-\mu) / m$, from previous comments.

The Pareto strategy of the firm is the same as in the noncooperative game, $\pi^{*}=\pi^{c}$. In fact, it is the well known maximum portfolio growth rule. Benefits are also proportional to wealth surplus, with a constant of proportionality that now depends on the weight $\mu$. It is worth noting that, for $\mu=1$ (i.e., when the common objective is to maximize the union's payoff) we recover the MPNE. Hence, we have proved that the MPNE, when $\alpha=\beta$ and $\gamma=\delta$, is Pareto efficient. This is a remarkable result that is really rare to find. The reason for having this property in the pension game is that the aim of maximizing an increasing and concave utility function of fund surplus is aligned with maximizing a utility from benefits collected from the fund surplus. The larger the fund surplus, more ample possibilities exist to collect more benefits. We establish this discussion in the following proposition.

Proposition 2.3 The MPNE of the pension game (4), (6), (7), with $\alpha=\beta$ and $\gamma=\delta$ is Pareto efficient.

Compared with the Pareto solutions for $\mu<1$, the MPNE over collect benefits. This is a typical situation known as "the tragedy of the commons": in general, cooperation leads to less aggressive policies of the players that, in this concrete model, allows a higher expected rate of growth of the fund surplus, thereby allowing higher benefit levels to be collected. Note that for $\alpha=\beta, \gamma=\delta, \mu<1$ and $x>0$

$$
\begin{aligned}
P^{*}(x) & =\frac{1-\gamma}{\gamma}\left(\frac{\rho}{1-\gamma}-r-\frac{1}{2 \gamma} \theta^{\top} \theta\right) x \\
& >\frac{1-\gamma}{\gamma}\left(\frac{\rho}{1-\gamma}-r-\frac{1}{2 \gamma} \theta^{\top} \theta-\frac{1-\mu}{1-\gamma} K^{-1}\right) x=P^{c}(x) .
\end{aligned}
$$

As a consequence, both players are better in the cooperative solution, as we will prove below. The payoff of the union with Pareto strategies is $J_{U}\left(x ; P^{c}, \pi^{c}\right)=J_{\mu=1}\left(x ; P^{c}, \pi^{c}\right)$, which coincides with $W(x)$ when $\mu=1$. From (8) and (23), constant $K$ for $\mu=1$ is $K=\left(\frac{1-\gamma}{\gamma}\left(\frac{\rho}{1-\gamma}-r-\frac{\theta^{\top} \theta}{2 \gamma}\right)\right)^{-\gamma}=$ 
$A$. Note that we are assuming $\alpha=\beta$ and $\gamma=\delta$. Analogously, the payoff of the firm with Pareto strategies is $J_{F}\left(x ; P^{c}, \pi^{c}\right)=J_{\mu=0}\left(x ; P^{c}, \pi^{c}\right)$, which coincides with $W(x)$ when $\mu=0$. From (9) and (23), the inverse of constant $K$ for $\mu=0$ is $K^{-1}=(1-\gamma)\left(\frac{\rho}{1-\gamma}-r-\frac{\theta^{\top} \theta}{2 \gamma}\right)<\rho+\frac{\rho}{\gamma}-\frac{1-\gamma}{\gamma}\left(r+\frac{\theta^{\top} \theta}{2 \gamma}\right)=B^{-1}$, because $0<\gamma<1$ and $\rho>0$. Then we have proved:

$$
\begin{aligned}
& J_{U}\left(x ; P^{c}, \pi^{c}\right)=J_{U}\left(x ; P^{*}, \pi^{*}\right)=V_{U}(x), \\
& J_{F}\left(x ; P^{c}, \pi^{c}\right)>J_{F}\left(x ; P^{*}, \pi^{*}\right)=V_{F}(x) .
\end{aligned}
$$

Although superior in terms of outcome, Pareto strategies suffer from the drawback of not being stable, since the union has incentives to deviate from the Pareto solution - except when $\mu=1$ as said above-.

\section{A pension game of minimizing the probability of ruin}

Undoubtedly, the firm's objective of maximizing an increasing utility of the fund surplus also cares about the health of the pension plan, but it is not the only sensible way to model the aim of the firm of honoring the comprised liabilities. Hence, let us study here a variant of the previous game. The objective of the union continues to be the same, as well as the dynamics. Only the objective of the firm changes. We suppose that, instead of maximizing the discounted expected utility of the fund surplus, it seeks to minimize the probability of ruin. More precisely, the firm fixes two fund surplus levels, $\ell>0$ (for the lower level) and $v>\ell$ (for the upper level), and starting the funding process with $X(0)=x \in(\ell, v)$, wishes to maximize the probability of reaching $v$ before $\ell$. Thus, $\ell$ is considered by the firm as a ruin point. Thus, the objective is different from the one given in the pension game in Section 2. There, the firm's aim was to maximize the expected growth rate of the pension fund, weighted by the risk aversion index of the firm. Here, the firm is concerned not only with growth, but also with preventing that the fund assets from reaching such a low level that is considered a ruin point. In other words, the firm wants to minimize the probability that the fund leaves the surplus region, so that solvency is guaranteed. Hence, the firm sets a surplus level, still positive, but so small, that below this threshold warning alarms are set off, as the insolvency region is close. The aim of the firm is to minimize the probability of reaching this "ruin point".

\subsection{Players' Payoffs}

We first deal with the payoffs of the firm. Given $P \in \mathcal{A}_{U}$ fixed, the firm chooses $\pi \in \mathcal{A}_{F}$ to maximize the probability that the fund surplus reaches $v$ before $\ell$, hence the payoff is

$$
J_{F}(x ; P, \pi)=\mathbb{P}\left(T_{v}<T_{\ell} \mid X(0)=x\right), \quad \ell<x<v .
$$


Here $T_{z}$ denotes the first time that $X$ hits the value $z \geq 0$. If $X^{(P, \pi)}$ is the fund surplus under profile $(P, \pi)$ satisfying (4), then $T_{z}=\inf \{t>0$ : $\left.X^{(P, \pi)}(t)=z\right\}$. We can rewrite the payoff as

$$
J_{F}(x ; P, \pi)=\mathbb{E}_{x} h\left(X^{(P, \pi)}(T)\right)
$$

with $T=\min \left\{T_{\ell}, T_{v}\right.$ )and $h(\ell)=0, h(v)=1$.

The union's payoff should be

$$
J_{U}(x ; P, \pi)=\mathbb{E}_{x}\left(\int_{0}^{T} e^{-\alpha t} u(P(t)) d t+e^{-\alpha T} g\left(X^{(P, \pi)}(T)\right)\right),
$$

where $g$ is a bequest function. These two functionals, together with the evolution of $X$ given in (4), specify the game. The game terminates at the random time $T$. Stochastic optimal control problems of this type are usually difficult to solve, explicitly or even numerically. Here, the problem seems even harder since we are dealing with a game. The problem arises because the value functions of the players satisfy a two-point boundary value system of differential equations. The boundary conditions are

$$
\begin{aligned}
& V_{U}(\ell)=g(\ell), \quad V_{U}(v)=g(v), \\
& V_{F}(\ell)=0, \quad V_{F}(v)=1 .
\end{aligned}
$$

The HJB system is ${ }^{3}$

$$
\begin{aligned}
\alpha V_{U}(x) & =\max _{P}\left\{\frac{P^{1-\gamma}}{1-\gamma}+\left(r x+\pi^{\top}(b-r \overline{1})-P\right) V_{U}^{\prime}(x)\right\}+\frac{1}{2} \pi^{\top} \Sigma \pi V_{U}^{\prime \prime}(x), \\
0 & =\max _{\pi}\left\{\left(r x+\pi^{\top}(b-r \overline{1})-P\right) V_{F}^{\prime}(x)+\frac{1}{2} \pi^{\top} \Sigma \pi V_{F}^{\prime \prime}(x)\right\} .
\end{aligned}
$$

Note that the maximizers obey the same rule as in the previous game of Section 2

$$
\begin{aligned}
& P=\left(V_{U}^{\prime}(x)\right)^{-1 / \gamma}, \\
& \pi=-\Sigma^{-1}(b-r \overline{1}) \frac{V_{F}^{\prime}(x)}{V_{F}^{\prime \prime}(x)} .
\end{aligned}
$$

and substituting them into the system above, we get

$$
\left\{\begin{aligned}
\alpha V_{U}(x) & =\frac{\gamma}{1-\gamma}\left(V_{U}^{\prime}(x)\right)^{1-1 / \gamma}+r x V_{U}^{\prime}(x)-\theta^{\top} \theta \frac{V_{F}^{\prime}(x)}{V_{F}^{\prime \prime}(x)} V_{U}^{\prime}(x)+\frac{1}{2} \theta^{\top} \theta\left(\frac{V_{F}^{\prime}(x)}{V_{F}^{\prime \prime}(x)}\right)^{2} V_{U}^{\prime \prime}(x), \\
0 & =r x V_{F}^{\prime}(x)-\frac{1}{2} \theta^{\top} \theta \frac{\left(V_{F}^{\prime}(x)\right)^{2}}{V_{F}^{\prime \prime}(x)}-\left(V_{U}^{\prime}(x)\right)^{-1 / \gamma} V_{F}^{\prime}(x) .
\end{aligned}\right.
$$

\footnotetext{
${ }^{3}$ The stochastic control problems of Dirichlet type, as in the problem considered here, have been analyzed with the dynamic programming approach in Krylov (1980). General results in the portfolio selection have been obtained in Browne (1997, 1999). JosaFombellida and Rincón-Zapatero (2006) introduced and studied these types of problems in pension funding.
} 
The boundary conditions are, as we said above,

$$
\begin{aligned}
& V_{U}(\ell)=g(\ell), \quad V_{U}(v)=g(v), \\
& V_{F}(\ell)=0, \quad V_{F}(v)=1 .
\end{aligned}
$$

We have not been able to find an explicit solution to this problem, even for simple selections of the bequest function $g$. Thus, we proceed to simplify the game. We will solve instead a modified game, where the union acts rather myopically, as if the planning horizon were not random, but fixed and of infinite horizon. If the upper value $v$ is large, then the probability that the process $X$ will leave the interval $(\ell, v)$ by the upper boundary may be made small. Moreover, as we will prove below, at the Nash equilibrium with suitable assumptions concerning the game's parameters, the lower boundary $\ell$ is never reached with positive probability. Thus, for large values of $v$, to approximate the union's payoff with the expected utility on the interval $[0, \infty)$ could be justified. Based on these considerations, we assume that the union wishes to maximize

$$
J_{U}(x ; P, \pi)=\mathbb{E}_{x} \int_{0}^{\infty} e^{-\alpha t} u(P(t)) d t,
$$

as in the previous sections, and analyze the game as an approximation to the true game, for which we do not know the solution. Now, the boundary conditions for the value function $V_{U}$ does not apply and we can solve the game explicitly. Then the boundary conditions that we impose are

$$
\begin{aligned}
& \lim _{t \rightarrow \infty} e^{-\alpha t} \mathbb{E}_{x}\left(V_{U}\left(X^{P, \pi}(t)\right)\right)=0, \\
& V_{F}(\ell)=0, \quad V_{F}(v)=1 .
\end{aligned}
$$

The first equality is the familiar transversality condition. The second set of conditions establishes that the probability of reaching $v$ before reaching $\ell$ is 0 at $x=\ell$ and 1 at $x=v$.

In the next proposition, we state the form of the MPNE of the pension game to minimize the probability of ruin.

Proposition 3.1 Assume that $\alpha>r$ and

$$
\alpha-r<\left(\frac{\gamma^{2}}{2}-\gamma+1\right) \theta^{\top} \theta .
$$

Then the value function of the union and the firm in the pension game (4), (30), (34) are

$$
\begin{aligned}
& V_{U}(x)=D \frac{x^{1-\gamma}}{1-\gamma}, \\
& V_{F}(x)=\frac{x^{1-\eta^{-}}-\ell^{1-\eta^{-}}}{v^{1-\eta^{-}}-\ell^{1-\eta^{-}}},
\end{aligned}
$$


respectively, where

$$
D=\left(r+\frac{1}{2 \eta^{-}} \theta^{\top} \theta\right)^{-\gamma}
$$

and

$$
\eta^{-}=\frac{\left(1-\frac{\gamma}{2}\right) \theta^{\top} \theta-\sqrt{\Delta}}{2(\alpha-r)},
$$

with $\Delta=\left(\left(1-\frac{\gamma}{2}\right) \theta^{\top} \theta\right)^{2}-2(\alpha-r) \gamma(1-\gamma) \theta^{\top} \theta$. The MPNE $\left(P^{r}, \pi^{r}\right)$ is given by

$$
\begin{aligned}
& P^{r}(x)=D^{-1 / \gamma} x, \\
& \pi^{r}(x)=\frac{1}{\eta^{-}} \Sigma^{-1}(b-r \overline{1}) x
\end{aligned}
$$

and the equilibrium fund surplus is a geometric Brownian motion process,

$$
d X^{*}(t)=\frac{\theta^{\top} \theta}{2 \eta^{-}} X^{*}(t) d t+\frac{1}{\eta^{-}} \theta^{\top} X^{*}(t) d w(t) .
$$

Proof. To solve the HJB system (33) and initial data (35) above we try solutions of the form $V_{U}(x)=D \frac{x^{1-\gamma}}{1-\gamma}$ and $V_{F}(x)=\frac{x^{1-\eta}-\ell^{1-\eta}}{v^{1-\eta}-\ell^{1-\eta}}$, where the constants $D$ and $\eta$ have to be determined. After plugging the guessed expressions for the value functions into the HJB system (33), we get that $D$ and $\eta$ have to satisfy

$$
\begin{aligned}
\frac{\alpha}{1-\gamma} & =\frac{\gamma}{1-\gamma} D^{-1 / \gamma}+r+\frac{1}{\eta} \theta^{\top} \theta-\frac{\gamma}{2 \eta^{2}} \theta^{\top} \theta, \\
0 & =r+\frac{1}{2 \eta} \theta^{\top} \theta-D^{-1 / \gamma} .
\end{aligned}
$$

Substituting $D^{-1 / \gamma}$ from the second identity into the first equation we get

$$
0=(\alpha-r) \eta^{2}-\left(1-\frac{\gamma}{2}\right) \theta^{\top} \theta \eta+\frac{1}{2} \gamma(1-\gamma) \theta^{\top} \theta,
$$

which admits the solutions $\eta^{ \pm}$given by $\eta^{-}=\frac{\left(1-\frac{\gamma}{2}\right) \theta^{\top} \theta-\sqrt{\Delta}}{2(\alpha-r)}$ and $\eta^{+}=$ $\frac{\left(1-\frac{\gamma}{2}\right) \theta^{\top} \theta+\sqrt{\Delta}}{2(\alpha-r)}$. Both are real, as the discriminant $\Delta$ of the equation (42) is positive under the assumptions of the proposition. Note that $\Delta>0$ if and only if $\alpha-r<h(\gamma) \theta^{\top} \theta$, where $h(\gamma)=\frac{\left(1-\frac{\gamma}{2}\right)^{2}}{2 \gamma(1-\gamma)}$, for $\gamma \in(0,1)$. It is not difficult to check that $\frac{\gamma^{2}}{2}-\gamma+1<1<h(\gamma)$, for all $\gamma \in(0,1)$. Thus, (36) implies $\Delta>0$. Now we prove that $0<\eta^{-}<1<\eta^{+}$. If $(1-\gamma / 2) \theta^{\top} \theta>2(\alpha-r)$ then $\eta^{+}>\frac{\left(1-\frac{\gamma}{2}\right) \theta^{\top} \theta}{2(\alpha-r)}>1$, because $\Delta>0$, and if $(1-\gamma / 2) \theta^{\top} \theta<2(\alpha-r)$ then $\eta^{+}>1$ if and only if $\sqrt{\Delta}>2(\alpha-r)-(1-\gamma / 2) \theta^{\top} \theta$. Taking the square we get (36). On the other hand, as $\sqrt{\Delta}<(1-\gamma / 2) \theta^{\top} \theta$ then 
$\eta^{-}>0$. To prove $\eta^{-}<1$, note that if $(1-\gamma / 2) \theta^{\top} \theta<2(\alpha-r)$, then $(1-\gamma / 2) \theta^{\top} \theta-\sqrt{\Delta}<2(\alpha-r)$, that is, $\eta^{-}<1$. And if $(1-\gamma / 2) \theta^{\top} \theta>2(\alpha-r)$, then $\eta^{-}<1$ if and only if $\sqrt{\Delta}>-2(\alpha-r)+(1-\gamma / 2) \theta^{\top} \theta$. Taking the square we get again (36). Thus $\eta^{-}$is the unique root of $(42)$ in $(0,1)$. The coefficient $D$ is well defined and positive. Moreover, $V_{U}$ defined in (37) is strictly convex and $V_{F}$, defined in (38), is strictly concave, because $0<\eta^{-}<1$. Now, the equilibrium candidate (39) and (40) is obtained from the optimality conditions (31) and (32), once $V_{U}, V_{F}$ are substituted. To see that the pair $\left(P^{r}, \pi^{r}\right)$ is indeed an MPNE, note that $V_{F}^{\prime \prime}<0$, because $0<\eta^{-}<1$, thus $\pi^{r}$ is a true maximizer of the HJB equation for the firm-it was already clear that $P^{r}$ was a maximizer of the HJB equation of the union-. Upon substitution of $\left(P^{r}, \pi^{r}\right)$ into the SDE (4), we get $(41)$, thus $\left(P^{r}, \pi^{r}\right)$ is clearly an admissible pair of strategies. Finally, we prove that the transversality condition (35) holds. See, as in the proofs of previous propositions, Hernández-Lerma (2004) Section 7, or Theorem 8.5 of Dockner et al (2000). By Arnold (1974), p. 139, for any real number $a$,

$$
\mathbb{E}_{x}\left(X^{*}(t)\right)^{a}=x^{a} \exp \left\{a\left(\frac{1}{2 \eta^{-}} \theta^{\top} \theta-\frac{1}{2\left(\eta^{-}\right)^{2}} \theta^{\top} \theta\right) t+\frac{a^{2}}{2} \frac{\theta^{\top} \theta}{\left(\eta^{-}\right)^{2}} t\right\} .
$$

After replacing $a$ by $1-\gamma$, we see that (35) holds if and only if

$$
\frac{(1-\gamma)}{2\left(\eta^{-}\right)^{2}} \theta^{\top} \theta\left(\eta^{-}-\gamma\right)<\alpha
$$

This condition is equivalent to

$$
\frac{1}{2} \theta^{\top} \theta \eta^{-}+r\left(\eta^{-}\right)^{2}>0,
$$

by (42). The left side, that coincides with $\left(\eta^{-}\right)^{2} D^{-1 / \gamma}$, is positive because $D>0,0<\eta^{-}<1$.

Remark 3.1 When $\alpha-r=\left(\frac{\gamma^{2}}{2}-\gamma+1\right) \theta^{\top} \theta$, then the previous proposition is true letting $\eta^{-}=1$ and $V_{F}(x)=\frac{\ln x-\ln \ell}{\ln v-\ln \ell}$.

As in the previous game, the equilibrium strategies $P^{r}$ and $\pi^{r}$ are proportional to the surplus $X$. It is interesting to note that they do not depend on $\ell$ or $v$. We have carried out a sensitivity analysis, as in Section 2.3.1, obtaining a similar response of the coefficient strategies to changes in the parameters, so we do not report our findings here. What we will do instead is a brief comparison of the equilibrium of the two games analyzed above, which are shown in the table below.

In what follows, note that the constant $\eta^{-}$, defined in Proposition 3.1, depends on the characteristics of the financial market and on the risk attitude of the union. 
Table 1: Equilibrium strategies

\begin{tabular}{lll} 
Game & Investment & Benefit \\
\hline Maximizing utility & $\pi^{*}(x)=\frac{1}{\delta} \Sigma^{-1}(b-r \overline{1}) x$ & $P^{*}(x)=A^{-1 / \gamma} x$ \\
Minimizing probability of ruin & $\pi^{r}(x)=\frac{1}{\eta^{-}} \Sigma^{-1}(b-r \overline{1}) x$ & $P^{r}(x)=D^{-1 / \gamma} x$
\end{tabular}

- Investment is equal in both games if the risk aversion parameter of the firm $\delta=\eta^{-}$. A higher (lower) risk aversion parameter $\delta$ leads to a more (less) cautious investment strategy in the maximizing-utilitygame than in the minimizing-the-probability-of-ruin game.

- Benefits comparison is not so straightforward. The share claimed by the union in the first scenario game is greater than in the second scenario game if and only if

$$
\alpha>r+\left((1-\gamma)\left(\frac{1}{\delta}-\frac{\gamma}{2 \delta^{2}}\right)+\frac{\gamma}{2 \eta^{-}}\right) \theta^{\top} \theta
$$

Since the r.h.s. tends to $-\infty$ as $\delta \rightarrow 0^{+}$, it is clear that the inequality is fulfilled (the situation now is that the rest of the parameters are fixed, and we look for values of the risk aversion parameter of the firm that satisfy the inequality). It is not difficult to prove ${ }^{4}$ that $\delta=\eta^{-}$ is the threshold such that the inequality above is fulfilled if and only if $\delta \in\left(0, \eta^{-}\right)$. It is worth comparing $P^{*}$ with $P^{r}$. Since $P^{*}(x)$ is a decreasing function of $\delta$ in $(0, \gamma)$ and increasing in $(\gamma, 1)$, it holds that for $\delta=\gamma, P^{*}(x)$ reaches its minimum value. Then, using (36), we obtain $P^{*}(x)<P^{r}(x)$ when $\delta \rightarrow 1$, and then $P^{*}(x)<P^{r}(x)$ for $\delta \in[\gamma, 1)$. This implies $\delta=\eta^{-} \in(0, \gamma)$.

Summing up, both firms' investment in risky assets and extra benefits claimed by the union are smaller in the minimizing-the-probability-of-ruin game than in the maximizing-utility-game when the risk aversion coefficient of the firm is below $\eta^{-}$. The situation is reversed for higher risk aversion coefficients. When $\delta=\eta^{-}$, both games share the same equilibrium strategies of benefits and investment.

\footnotetext{
${ }^{4}$ Equating expressions for $A$ and $D$, this is equivalent to

$$
(\alpha-r)\left(\eta^{-}\right)^{2}-\left(1-\frac{\gamma}{2}\right) \theta^{\top} \theta \eta^{-}+\frac{1}{2} \gamma(1-\gamma) \theta^{\top} \theta-\frac{\gamma}{2 \eta^{-}} \theta^{\top} \theta \delta^{2}+\frac{\gamma}{2} \theta^{\top} \theta \delta=0,
$$
}

that is to say $\delta=\eta^{-}$, by (42). 


\section{Conclusions}

In the large body of literature about defined benefit pension plans, the interaction between sponsor and participants has been considered as one of a sole decision agent. The participants took a passive role. Our starting point in this paper has been that, at least when the fund is overfunded, the fund surplus could be given a strategic role by incrementing the benefits of the participants at the time of retirement. We have proposed an asymmetric noncooperative game in two different scenarios that allows for analytical solutions and that could be easily implemented in real pension plans of defined benefits type, since the Nash equilibrium is linear. We have shown that players with the same relative risk aversion index will seek noncooperatively a cooperative outcome, or in other words, the decentralized interaction gives rise to an efficient split of the fund surplus. Moreover, if the sponsoring firm is worried about the safety of the pension plan, this behavior corresponds in equilibrium with a firm that wants to maximize its own discounted expected utility in the first scenario game. Further research will be directed at analyzing hierarchical interaction between firm and participants, which is an interesting situation if the two players have different negotiating power. Here, the relevant solution concept is the Stackelberg equilibrium. It would be worth comparing the Nash equilibrium with the Stackelberg equilibrium to see to what extent the advantage of being the leader, which is a usual property, is maintained in this case.

\section{Acknowledgements}

Support from the Ministerio de Economía y Competitividad (Spain), grants ECO 2014-56384-P, MDM 2014-0431, and Comunidad de Madrid, MadEco-CM S2015/HUM-3444 is gratefully acknowledged.

\section{References}

Arnold, L., 1974. Stochastic Differential Equations. Theory and Applications. John Wiley and Sons, New York.

Browne, S., 1997. Survival and growth with a fixed liability: Optimal portfolios in continuous time. Mathematics of Operations Research 22, 468-493.

Browne, S., 1999. Beating a moving target: Optimal portfolio strategies for outperforming a stochastic benchmark. Finance and Stochastics 3, 275-294.

Browne, S., 2000. Stochastic differential portfolio games. Journal of Applied Probability $37,126-147$. 
Cabo, F., García-González, A., 2014. The endogenous determination of retirement age and social security benefits. Macroeconomics Dynamics 18, 93-113.

Cairns, A.J.G., 2000. Some notes on the dynamics and optimal control of stochastic pension fund models in continuous time. Astin Bulletin 30, 19-55.

Chang, S.C., 1999. Optimal pension funding through dynamic simulations: the case of Taiwan public employees retirement system. Insurance: Mathematics and Economics 24, 187-199.

Chang, S.C., Tsai, C.H., Tien, C.J., Tu, C.Y., 2002. Dynamic funding and investment strategy for defined benefit pension schemes: a model incorporating asset-liability matching criteria. Journal of Actuarial Practice 10, $131-153$.

Chang, S.C., Tzeng, L.Y., Miao, J.C.Y., 2003. Pension funding incorporating downside risks. Insurance: Mathematics and Economics 32, 217-228.

Delong, L., Gerrard, R., Haberman, S., 2008. Mean-variance optimization problems for an accumulation phase in a defined benefit plan. Insurance: Mathematics and Economics 42, 107-118.

Dockner, E., Jørgensen, S., Van Long, N., Sorger, G. 2000. Differential games in economics and management sciences. Cambridge University Press, Cambridge, UK.

Guan, G., Liang, Z., 2016. A stochastic Nash equilibrium portfolio game between two DC pension funds. Insurance: Mathematics and Economics 70, $237-244$.

Haberman, S., Butt, Z., Megaloudi, C., 2000. Contribution and solvency risk in a defined benefit pension scheme. Insurance: Mathematics and Economics $27,237-259$.

Haberman, S., Sung, J.H., 1994. Dynamics approaches to pension funding. Insurance: Mathematics and Economics 15, 151-162.

Hainaut, D., 2014. Impulse control of pension fund contributions, in a regime switching economy. European Journal of Operational Research 239, 810-819.

Hainaut, D., Deelstra, G., 2011. Optimal funding of defined benefit pension plans. Journal of Pension Economics and Finance 10, 31-52. 
Hernández-Lerma, O., 1994. Lectures On Continuous-Time Markov Control Processes. Sociedad Matemática Mexicana. México D.F.

Huang, H.C., Cairns, A.J.G., 2006. On the control of defined-benefit pension plans. Insurance: Mathematics and Economics 38, 113-131.

Josa-Fombellida, R., Rincón-Zapatero, J.P., 2001. Minimization of risks in pension funding by means of contribution and portfolio selection. Insurance: Mathematics and Economics 29, 35-45.

Josa-Fombellida, R., Rincón-Zapatero, J.P., 2004. Optimal risk management in defined benefit stochastic pension funds. Insurance: Mathematics and Economics 34, 489-503.

Josa-Fombellida, R., Rincón-Zapatero, J.P., 2006. Optimal investment decisions with a liability: The case of defined benefit pension plans. Insurance: Mathematics and Economics 39, 81-98.

Josa-Fombellida, R., Rincón-Zapatero, J.P., 2010. Optimal asset allocation for aggregated defined benefit pension funds with stochastic interest rates. European Journal of Operational Research 201, 211-221.

Josa-Fombellida R., Rincón-Zapatero, J.P., 2012. Stochastic pension funding when the benefit and the risky asset follow jump diffusion processes. European Journal of Operational Research 220, 404-413.

Krylov, N.V., 1980. Controlled Diffusion Processes. Springer, New York.

Lancaster, K., 1973. The dynamic inefficiency of capitalism. Journal of Political Economy 81, 10921109.

Le Courtois, O., Menoncin, F., 2015. Portfolio optimisation with jumps: Illustration with a pension accumulation scheme. Journal of Banking and Finance 60, 127-137.

Leong, C.K., Huang, W., 2010. A stochastic differential game of capitalism. Journal of Mathematical Economics 46, 552-561.

Merton, R.C., 1971. Optimal consumption and portfolio rules in a continuoustime model. Journal of Economic Theory 3, 373-413.

Merton, R.C., 1990. Continuous Time Finance. Blackwell, MA.

Xu, J., Kannan, D., Zhang, B., 2007. Optimal dynamic control for the defined benefit pension plans with stochastic benefit outgo. Stochastic Analysis and Applications 25, 201-236. 